\title{
Assessment of gonadal maturation by evaluation of spermaturia
}

\author{
Franz Schaefer, Joachim Marr, Christoffer Seidel, Wolfgang Tilgen, Karl Schärer
}

\begin{abstract}
The urinary excretion of spermatozoa (spermaturia) reflects the achievement of exocrine testis function during male puberty. In order to test the sensitivity and practicability of repetitive urine sampling, we analysed the sediments of 1160 first morning urine specimens obtained on successive days from 129 healthy schoolboys aged $10 \cdot 1$ to $17 \cdot 8$ years for the presence of spermatozoa. The proportion of subjects with sperm positive urines increased from pubic hair stage (PH) $1(6 \%)$ to PH $5(92 \%)$ with a steep rise between PH 2 and 3. Estimated median age of first positive spermaturia ('spermarche') was $14 \cdot 1$ years. While at PH 1 to 4 all positive samples were found within the first five days of collection, at PH 5 cumulative frequency of spermaturia increased up to day 8 . We conclude that repetitive morning urine sampling is a useful tool in assessing spermaturia and may be helpful to screen for testicular damage in epidemiological surveys.
\end{abstract}

The detection of spermatozoa in the urine has been proposed as a quick, non-invasive method to assess the functional state of the maturing gonad and may be useful in screening for spermatogenic damage in male adolescents. ${ }^{1-6}$ The evidence derived from longitudinal ${ }^{245}$ and cross sectional $^{3}$ studies that spermaturia is a discontinuous phenomenon seems, however, to limit its value as a routine screening procedure. In those earlier reports either a single 24 hour urine $^{25}$ or variable numbers of morning urine specimens ${ }^{4} 6$ were analysed. Considering the intermittent appearance of spermaturia, which probably occurs as a urethral wash out phenomenon after involuntary or masturbatory sperm emission, ${ }^{7}$ the probability of urinary sperm excretion should rise according to the number of specimens collected. No systematic investigations have been performed, however, in study populations of adequate size over extended

Table 1 Age distribution of the 129 boys under study. Age ranged from $10 \cdot 1$ to $17 \cdot 8$ (median $12 \cdot 8$ ) years

\begin{tabular}{|c|c|c|c|c|c|c|c|}
\hline & \multicolumn{7}{|c|}{ Age (years) } \\
\hline & 10 & 11 & 12 & 13 & 14 & 15 & $16-17$ \\
\hline No of boys & 14 & 26 & 31 & 14 & 20 & 18 & 6 \\
\hline
\end{tabular}

periods of time so far. Therefore, we attempted to determine the sensitivity and practicability of repetitive morning urine collections in a group of 129 healthy peripubertal boys, and to relate spermaturia to their state of sexual maturation.

\section{Subjects and methods}

One hundred and twenty nine healthy boys from five secondary schools in the city of Heidelberg agreed to participate in the study. The distribution of age and stages of puberty are given in tables 1 and 2. The children and their parents were informed that the purpose of the study was to evaluate normal sexual maturation by clinical assessment and urinary hormone excretion; however, they were not aware that spermaturia was investigated.

The boys were requested to collect their first morning void for two consecutive weeks from Monday to Friday respectively. In case of night time micturition they were advised to collect the first nocturnal urine. The specimens were brought to school in $100 \mathrm{ml}$ plastic vessels and collected daily by one of the investigators. The bottles contained $3 \mathrm{ml}$ of a $0.7 \%$ saponin solution in $0.4 \%$ aqueous formalin. The urine was stored at $4^{\circ} \mathrm{C}$ and analysed within 10 days after collection.

Urine analysis was performed according to the method described by Hirsch et al. ${ }^{3}$ In brief, a $10 \mathrm{ml}$ aliquot of the well shaken urine was centrifuged at $1800 \mathrm{~g}$ for 15 minutes. The pellet was resuspended in $0.5 \mathrm{ml}$ of the supernatant after decantation. The resuspended sediment was examined microscopically. Forty visual fields were checked at a magnification of $\times 400$ for the presence of spermatozoa. The same observer (JM) investigated all specimens. A specimen was considered sperm positive if one or more spermatozoa were detected.

At the end of the first week of urine collection, a clinical examination of the pubertal status was performed by a single observer (JM). Genital and pubic hair stage was recorded according to Tanner. ${ }^{8}$ The volume of each testicle was estimated by use of a Prader orchidometer, and the mean volume of the two testes was calculated.

ANALYSIS OF DATA

As not all boys delivered complete series of 10

Table 2 Distribution of Tanner stages of puberty $(n=129)$. PH=pubic hair stage, $G=$ genital stage

\begin{tabular}{lllllllllll}
\hline & PHI & $G 1$ & PH2 & G2 & PH3 & G3 & PH4 & G4 & PH5 & G5 \\
\hline No of boys & 34 & 41 & 45 & 27 & 16 & 14 & 17 & 14 & 17 & 33 \\
\hline
\end{tabular}


Table 3 Distribution of number of collected urines

\begin{tabular}{lc}
\hline No of urine specimens collected & $N o(\%)$ of subjects \\
\hline 4 & $2(1 \cdot 6)$ \\
5 & $6(4 \cdot 7)$ \\
6 & $2(1 \cdot 6)$ \\
8 & $5(3 \cdot 8)$ \\
9 & $10(7 \cdot 7)$ \\
10 & $104(80 \cdot 6)$ \\
& $129(100 \cdot 0)$ \\
\hline
\end{tabular}

morning urine specimens (table 3), the cumulative probability to remain sperm negative was calculated by use of the Kaplan-Meier life table analysis. ${ }^{9}$ The subjects who dropped out of the study before spermaturia was detected were classified as lost to follow up at the last day of collection and treated as censored data. Spermaturia rates were estimated stratified by age, pubertal stage, and testicular volume.

The median age at spermarche, defined as the age at which spermaturia can be demonstrated in $50 \%$ of a given population, was estimated by probit analysis. ${ }^{10}$ In this procedure, the percentage of subjects exhibiting spermaturia are recorded at the successive age stages. To this percentage distribution a probit transformation is applied, and a regression line fitted to the probit values. Thus it is possible to calculate the median and variance of age at spermarche, assuming a Gaussian distribution of the timing of the event in the population.

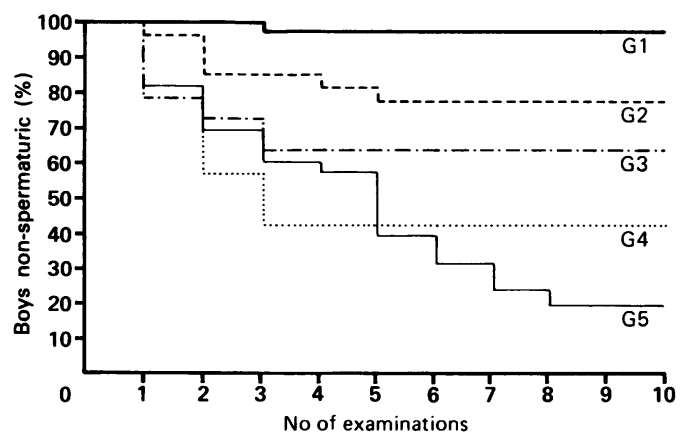

Figure 1 Kaplan-Meier estimation of probability to remain sperm negative in up to 10 consecutive morning urine samples, strattified by I'anner's genital $(G)$ stage.

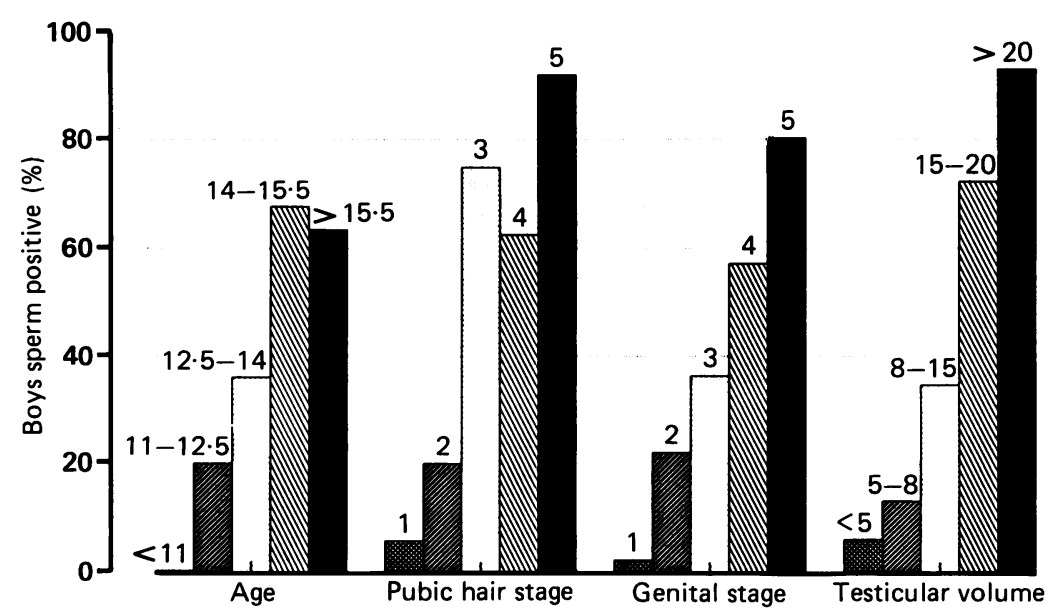

Figure 2 Overall cumulative probability of spermaturia according to age, Tanner stages for pubic hair (1-5) and genitals (1-5), and testicular volume $(m l)(n=129)$.
All calculations were performed by use of the respective procedures supplied in the SAS software package. ${ }^{11}$

\section{Results}

As shown in table 3, the overall participation rate in the study was very high. Almost $90 \%$ of the boys collected nine or 10 morning urines. A total of 1120 specimens was analysed.

The Kaplan-Meier analysis of all patients' probability to remain sperm free with increasing numbers of examinations is shown in fig 1 , stratified by Tanner's genital (G) stage. The proportion of sperm positive subjects increased with the state of genital maturity. After 10 urine collections, less than $20 \%$ of the boys in G5 had not exhibited spermaturia in any specimen, whereas in G1 spermatozoa were only found in one out of 40 boys. In presence of stages $\mathrm{Gl}$ to G4, no new sperm positive individuals were detected beyond day 5, whereas in G5 the cumulative frequency of spermaturia gradually increased up to day 8 .

Similar analyses were performed according to the other maturational parameters under study. A survey of the probability of spermaturia as calculated by Kaplan-Meier estimation is given for age, pubertal stages, and testicular volume in fig 2. Age and testicular volume were subdivided into five classes. No subject was found sperm positive below 11 years of age; in the upper age groups, only about two thirds of the boys exhibited spermaturia. When related to pubic hair $(\mathrm{PH})$ stage, a steep rise of the sperm positive fraction was seen between $\mathrm{PH} 2$ and PH3, with more than $90 \%$ being positive at PH5. The most discriminative parameter was testicular volume; when the mean testicular size exceeded $20 \mathrm{ml}, 93.5 \%$ of the subjects excreted urinary sperm.

The probit analysis for the estimation of the age at spermarche is depicted in fig 3 . The median age at spermarche was calculated as $14 \cdot 1$ years, with a $95 \%$ confidence interval of 13.5 to 14.9 years.

We also performed a quantitative analysis of spermaturia. The number of positive morning urine specimens per subject ranged from one to nine. A wide variation of sperm concentration (one to 458 spermatozoa/40 visual fields) was noted. In the sperm positive individuals no

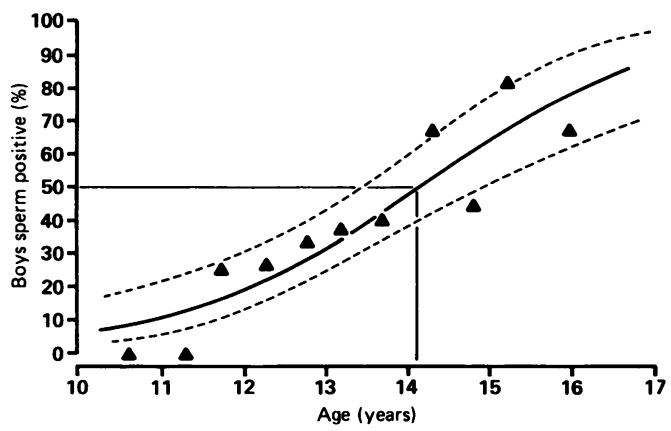

Figure 3 Probit analysis of median age at spermarche. Age was grouped in six month intervals. Triangles mark unsmoothed percentages of sperm positive individuals per age group. Full line indicates smoothed sigmoid probability curve, dotted lines $95 \%$ confidence intervals. 
significant correlation was found between the number of spermatozoa per $\mathbf{4 0}$ visual fields and age $(r=0.09, p=0.52)$ or testicular volume $(r=0.23, p=0.12)$. The correlations were not improved when the sperm count was related to urinary creatinine concentration to account for the varying dilution of the morning urines.

\section{Discussion}

The primary target of our study was to evaluate how regularly spermaturia can be picked up in healthy boys by the consecutive collection of 10 morning urines. In contrast to earlier studies ${ }^{3} 4$ the good compliance of the study participants allowed us to calculate overall probability rates from appropriate numbers of boys at all stages of puberty.

Our data demonstrate that spermaturia is better predicted by the clinical stage of puberty than by chronological age. The most powerful criterion to be sperm positive was a mean testicular volume of greater than $20 \mathrm{ml}$, but also in Tanner PH stage 5 more than $90 \%$ of the boys exhibited spermaturia (fig 2). Thus it is evident that spermaturia is detectable in the large majority of sexually mature subjects within a 10 day period of observation. Detailed analysis of cumulative spermaturia rates according to the number of investigated urines indicates that, however, once spermarche has occurred, spermaturia is a more regular event during early and mid puberty than in grown up individuals, where the first spermatozoa sometimes were not detected before the eighth day of collection (fig 1). This finding confirms an earlier suggestion by Hirsch et al who, analysing a maximum of five consecutive morning urine specimens, found a more slowly rising cumulative probability of spermaturia in 16 year olds compared with younger boys. ${ }^{3} \mathrm{We}$ assume that this difference may reflect changes from predominantly spontaneous nocturnal sperm emission towards rather intermittent masturbatory ejaculations in late puberty.

The high sperm detection rate in mature boys with the sampling protocol used in this study also indicates that a $\mathbf{1 0}$ day repetitive morning urine collection is more suitable to identify reliably postspermarcheal subjects than a 24 hour or a short term morning urine sampling regime as proposed in earlier reports. ${ }^{235}$

Despite the high sensitivity of our sampling technique it probably does not allow quantitative estimations of sperm production during puberty. In early studies of biopsy specimens a gradual increase of the density of mature spermatozoa in the tubular lumen during adolescence has been demonstrated, ${ }^{12}$ and immediate postejaculatory urinary sperm concentrations correlated with ejaculate sperm counts. ${ }^{7}$ Thus one might expect that the sperm number observed in the urine sediment should increase with advancing puberty. Yet no such correlation was found, possibly due to previous voiding between ejaculation and urine collection, variable urine volume and concentration, or other factors.

The median 'age at spermarche' observed in this cross sectional study was $14 \cdot 1$ years. In relation to pubertal stage, the $50 \%$ mark was between PH 2 and 3, although in a few cases spermaturia was found even in clinically prepubertal boys. Despite methodological and ethnic differences these results compare well with the findings of Richardson and Short who found a median age at spermarche of $13 \cdot 3$ years, ${ }^{2}$ Hirsch et al (14.5 years), ${ }^{3}$ and .Nielsen et al $(13.4$ years). ${ }^{5}$ It is also noteworthy that the median age at spermarche found in these studies corresponds to the age at first conscious ejaculation as evaluated by interviews. ${ }^{13}$

In summary, repetitive assessment of spermaturia is a sensitive, ethically acceptable, noninvasive procedure to determine the state of spermatogenic activity in pubertal subjects. The method cannot replace detailed quantitative sperm analysis, but may be used as a qualitative screening test to investigate potential damage to the maturing testis. It appears also suitable for epidemiological investigations in precocious or delayed puberty, for patients having chemotherapy or irradiation treatment, or for those with other chronic diseases potentially adversely affecting the gonads. Results of such studies can be analysed using the methods and be compared with the normal data presented in this study.

The authors thank Professor Michael Preece, Institute for Child Health, London, for his advice in the statistical analysis of the material.

1 Baldwin B. The determination of sex maturation in boys by a laboratory method. F Comp Psychol 1928;8:39-43.

2 Richardson D, Short $R$. Time of onset of sperm production in boys. F Biosoc Sci 1978;5:15-25.

3 Hirsch M, Shemesh J, Modan M, Lunenfeld B. Emission of spermatozoa: age of onset. Int F Androl 1979:2:289-98.

4 Hirsch M, Lunenfeld B, Modan M, Ovadia J, S.289. Spermarche-the age of onset of sperm emission. 7 Adolesc Health Care 1985;6:35-9.

5 Nielsen CT, Skakkebaek NS, Richardson DW, et al. Onset of the release of spermatozoa (spermarche) in boys in relation to age, testicular growth, pubic hair, and height. $尹$ Clin Endocrinol Metab 1986;62:532-5.

6 Kulin HE, Frontera ME, Demers LD, Bartholomew MJ, Lloyd TA. The onset of sperm production in pubertal

7 Weissenberg $\mathbf{R}$, Rozenman H, Hova M, Modan M, Lunenfeld $B$. The diagnostic value of sperm in postejaculatory urine. Int $\mathcal{f}$ Androl 1984;7:142-8.

8 Tanner JM. Growth at adolescence. 2nd Ed. Oxford: Blackwell Scientific Publications, 1962.

9 Kaplan EL, Meier P. Nonparametric estimation from incomplete observations. Foumal of the American Statistical Association 1958;53:457-62.

10 Finney DJ. Probit analysis. London: Cambridge University Press, 1971.

11 SAS Institute Inc. SAS user's guide: statistics version 5. Cary, NC: SAS Institute, 1985.

12 Charny CW, Conston AS, Meranze DR. Development of the testis. Fertil Steril 1952;3:461-79.

13 Laron Z, Arad J, Gurewitz R, Grunebaum M, Dickerman Z. Age at first conscious ejaculation: a milestone in male puberty. Helv Paediatr Acta 1980;35:13-20. 